\title{
Surface Gravities and Masses in Substellar Objects
}

\author{
Subhanjoy Mohanty \\ Harvard-Smithsonian CfA, 60 Garden Street, Cambridge, MA 02138
}

Gibor Basri

Astronomy Department, University of California, Berkeley, CA 94720

Ray Jayawardhana

Astronomy Department, University of Michigan, Ann Arbor, MI 48109

\begin{abstract}
Using high-resolution optical spectra, we determine effective temperatures and gravities for a sample of very low-mass stellar and substellar PMS cluster objects. Masses and radii are then derived using known cluster distance and photometry; two of our targets seem to have planetary masses. Our results are independent of theoretical evolutionary tracks. While our results agree with the track predictions for hotter, higher mass objects, discrepancies appear for the coolest, lowest mass ones. This may be due to track uncertainties related to formation effects, and/or internal conditions, in these very young, ultra-low-mass objects.
\end{abstract}

\section{Method of Analysis}

Our data consists of high-resolution $(\mathrm{R} \approx 31000)$ optical spectra of mid- to late M-type PMS objects in Upper Scorpius and Taurus. We derive $T_{\text {eff }}$ and gravity $(\log g)$, from a fine analysis of of the $\mathrm{TiO}$ bandheads and $\mathrm{Na} \mathrm{I}$ and $\mathrm{K} \mathrm{I}$ alkali doublets, through comparison with the latest synthetic spectra (Allard et al. 2001). These spectral diagnostics complement each other, removing temperature/gravity degeneracies in the behavior of each; together they allow us to determine $\mathrm{T}_{\text {eff }}$ and $\log g$ to a precision of $\pm 50 \mathrm{~K}$ and \pm 0.25 dex respectively. Combining our values of $\mathrm{T}_{\mathrm{eff}}$ and $\log g$ with the known distance to Upper Scorpius and Taurus $(\approx 140 \mathrm{pc})$ and observed and synthetic photometry, we also derive masses (within a factor of 2 ) and radii (within 30\%). We emphasize that our derived quantities are independent of any theoretical evolutionary models. Finally, various tests indicate that our results are not significantly affected by photospheric dust, cool magnetic spots, or metallicity variations.

\section{Results}

Our temperature and gravity results are compared to the Lyon theoretical evolutionary tracks (Chabrier et al. 2000) in Fig. 1. For $T_{\text {eff }} \gtrsim 2750 \mathrm{~K}$, our gravities are consistent (within our errors) with the theoretical predictions for the ex- 


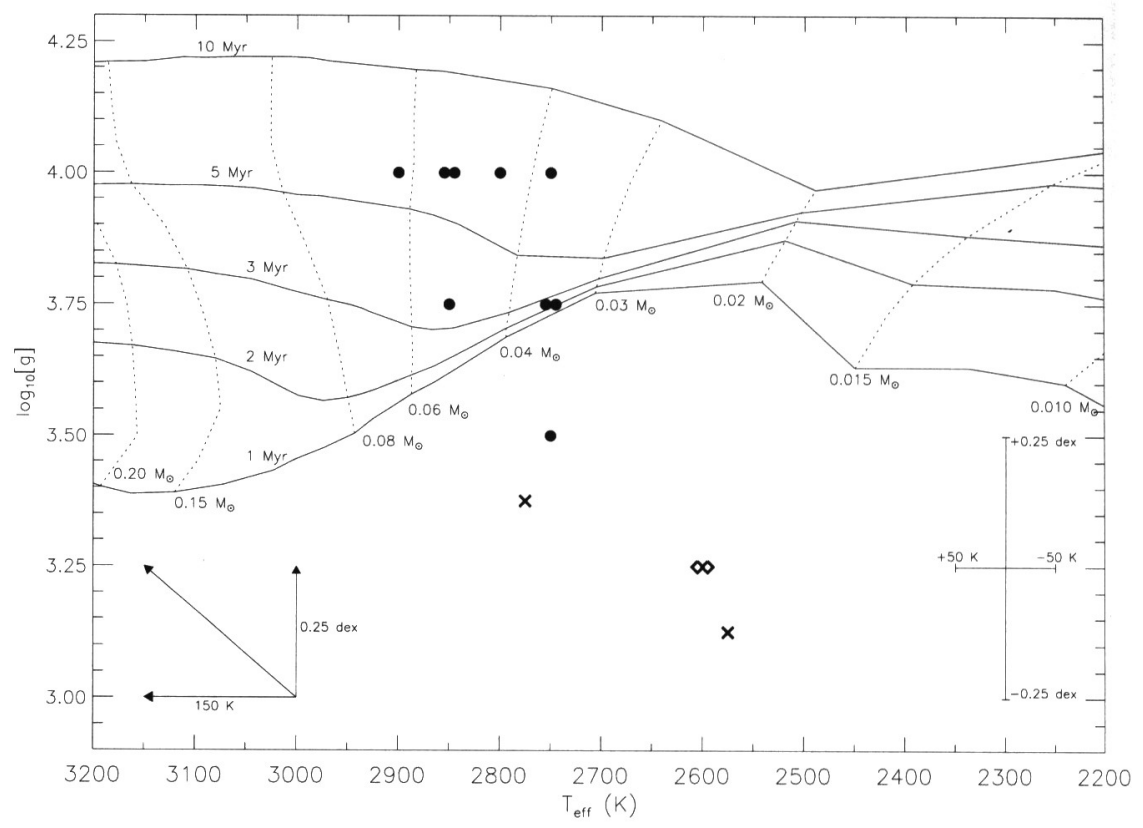

Figure 1. GG Tau Ba \& Bb are crosses, Usco $128 \& 130$ (both planetary mass) are diamonds, all other Upper Scorpius objects are filled circles. In this $\log g$ versus $\mathrm{T}_{\text {eff }}$ plot most objects agree well with the Lyon tracks, except $\mathrm{Bb}$ (cooler cross) and USco $128 \& 130$. Error bars for $T_{\text {eff }}$ and $\log g( \pm 50 \mathrm{~K}$, $\pm 0.25 \mathrm{dex})$ are shown; arrows show the largest possible shift in our values $(\leq 150 \mathrm{~K}, \leq 0.25 \mathrm{dex})$ from cool spots.

pected cluster ages (Upper Scorpius: $5 \pm 2 \mathrm{Myr}$; Taurus: $\sim 1 \mathrm{Myr}$ ). However, our $\log g$ values are much lower than predicted for our coolest targets; consequently, they appear much younger than expected. Using distance and photometry information, we then derive masses and radii. Most of our targets turn out to be very low-mass stars and brown dwarfs; two appear to have planetary masses of 6-7 $\mathrm{M}_{J}$, and are at most at the D-fusion minimum-mass boundary (Fig. 2). While most of our objects agree very well with the Lyon track predictions for mass versus radius, the lowest masses $\left(\mathrm{M} \lesssim 30 \mathrm{M}_{J}\right.$; these are the objects with lowest gravities in Fig. 1) have significantly larger radii than predicted for their expected ages (Fig. 2). Once again, just as in the $\mathrm{T}_{\text {eff }}$-gravity plane, they seem implausibly young $(\$ 0.5 \mathrm{Myr})$ when compared to the tracks.

Various lines of argument indicate that the age-spread in our sample suggested by the tracks is not real. Instead, we think it more likely that the evolutionary models have unresolved uncertainties at young ages for the lowest masses. These might arise from a variety of causes, such as accretion effects or non-isentropic internal structure; these issues must be addressed in the future. Our results also indicate that the faintest very young late-M objects may be much lower in mass (close to the D-burning limit) than previously suspected. Our detailed analysis can be found in Mohanty et al. (2003a,b). 


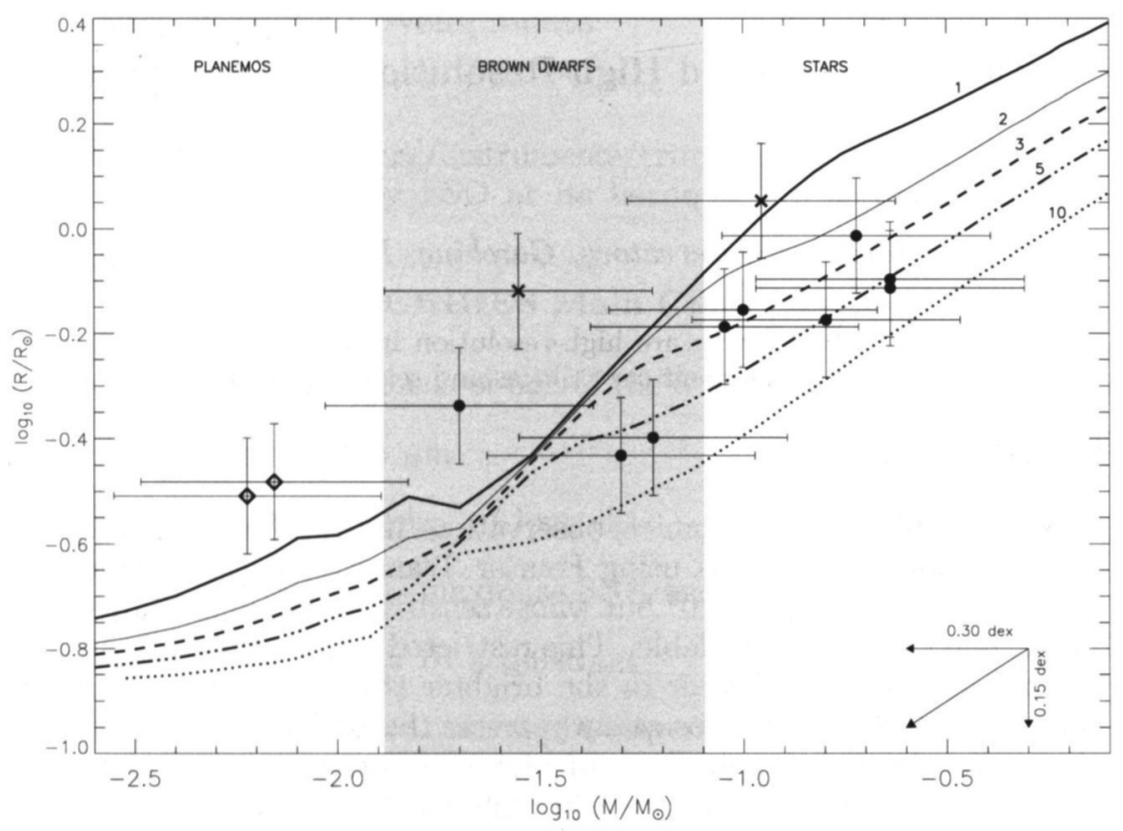

Figure 2. GG Tau Ba \& Bb are crosses, Usco 128 \& 130 (both planetary mass) are diamonds, all other Upper Scorpius objects are filled circles. In this radius $\left(\log \left[\mathrm{R} / \mathrm{R}_{\odot}\right]\right)$ versus mass $\left(\log \left[\mathrm{M} / \mathrm{M}_{\odot}\right]\right)$ plot, $\mathrm{GG} \mathrm{Tau} \mathrm{Bb}$ and USco 128 \& 130 are much larger than predicted; others agree reasonably with the tracks. Arrows show shift if any object were an equal-mass binary.

\section{References}

Allard, F., Hauchildt, P., Alexander, D., Tamanai, A., \& Schweitzer, A. 2001, ApJ, 556, 357

Chabrier, G., Baraffe, I., Allard, F., \& Hauschildt, P. 2000, ApJ, 542, 464

Mohanty, S., et al. 2003a, submitted

Mohanty, S., et al. 2003b, submitted 